\title{
Intelligent Fleet Management System for Open Pit Mine
}

\author{
Hajar BNOUACHIR ${ }^{1}$, Atae SEMMAR ${ }^{7}$ \\ Hicham MEDROMI \\ Engineering Research Laboratory (LRI), System \\ Architecture Team (EAS), National and High School of \\ Electricity and Mechanic (ENSEM), Hassan II University of \\ Casablanca, Research Foundation for Development and \\ Innovation in Science and Engineering, Casablanca, \\ Morocco \\ Meryiem CHERGUI ${ }^{2}$, Aziza CHAKIR ${ }^{5}$ \\ Engineering Research Laboratory (LRI), System \\ Architecture (EAS), National and High School of Electricity \\ and Team Mechanic (ENSEM), Hassan II University of \\ Casablanca, Casablanca, Morocco
}

\author{
Nadia MACHKOUR ${ }^{3}$, Mourad ZEGRARI ${ }^{4}$ \\ Structural Engineering, Intelligent Systems and Electrical \\ Energy Laboratory, Ecole Nationale Supérieure des Arts et \\ Métiers, Hassan II University of Casablanca, Innovation \\ Lab for Operations, Mohammed VI Polytechnic University \\ Benguerir, Morocco, Casablanca \\ Morocco
}

Laurent DESHAYES ${ }^{6}$

Innovation Lab for Operations Mohammed VI Polytechnic University

Benguerir, Morocco

\begin{abstract}
Fleet management systems are currently used to coordinate mobility and delivery services in a wide range of areas. However, their traditional control architecture becomes a critical bottleneck in open and dynamic environments, where scalability and autonomy are key factors in their success. In this article, we propose an intelligent distributed Fleet Management System architecture for an open pit mine that allows mining vehicles control in a real time context, according to users' requirements. Enriched by an intelligence layer made possible by the use of high-performance artificial intelligence algorithms and a reliable and efficient perception mechanism based on Internet of Things technologies and governed by an smart and integrated decision system that allows the fleet management system to improve its agility and its response to user requirements, our architecture presents numerous contributions to the domain. These contributions enable the fleet management system to meet the interoperability and autonomy requirements of the most widely used standards in the field, such as ISA 95.
\end{abstract}

Keywords-Fleet management system; open pit mine; monitoring; architectures; artificial intelligence; real time system

\section{INTRODUCTION}

Monitoring the company's activity in real time has many assets and advantages not to be ignored. This instant management allows us to visualize and know the real situation of the company as well as to monitor its objectives, key figures and performance indicators; it facilitates decision-making, manages its daily life more effectively and improves its reactivity and competitiveness.

Regardless the structure size, any good manager must be equipped with the appropriate resources to manage his activity effectively. This means that the positioning of carried out actions and committed budgets should be monitored, for both, quantitative and qualitative objectives.
The Fleet Management System (FMS) [1] is a modern technological solution to manage the fleet's vehicles in terms of automation and business process optimization and to link all business sectors such as dispatching, purchasing, communications, navigation, accounting and finance in a single system. FMS is easy to control and optimize, its foundations date back to the 1980s, when the computer was integrated in the vehicle and connected to different wireless networks by satellite and terrestrial [2].

In the mining sector, the main objective of the fleet management system is to optimize mine production and efficiency based on real-time data. More specifically, the FMS aims to maximize mine production, minimize stock handling, feed the pro-cessing plant at the planned rate and meet quality mixing constraints [3].

In this article, we will present first, fleet management systems' state of the art as well the use of artificial intelligence in it; we will also initiate a fleet management system evaluation model based on ISA 95. We will discuss after that, open pit mine FMS problems before proposing a general intelligent FMS architecture. We will end this article with conclusion and perspectives.

\section{STATE OF THE ART}

\section{A. Fleet Management Systems}

Generally, Fleet management system (FMS) is a term used to refer to a wide range of solutions for different fleet applications in the fields of transport, distribution and logistics [4] [5]. It includes targeted planning, supervision and control of fleet operation ac-cording to available transport resources and application constraints. The FMS aims to reduce risks, increase service quality and improve a fleet's operational efficiency while minimizing costs [6]. 
The implementation of operational research techniques as the main means of ad-dressing the high cost of transporting equipment to open-pit mines, has attracted re-searchers and laid the foundation for fleet management systems (FMS) used in open-pit mines.

They considered the FMS as a real time decision-making system [7] [8] for materials handling in an open pit mine. FMS obtains the required information about mining operations from the database and makes decisions accordingly. These decisions are then implemented in the operation and the FMS is recalled whenever a new decision is required.

Fig. 1 shows the communication between open-pit mining and the FMS.

The modular mining system revealed models [10] [11]and algorithms, namely:

- Shortest path: It aims at optimizing travel time using the Dijkstra method [12] [3], its advantages are that the algorithm does not need to study all contours and it has an order of $\mathrm{n} 2$ so that it is sufficiently efficient to use for relatively large applications. But the model takes a long time, it has failures in case of negative edges.

- Allocation planning[13]: Minimizes the total number of trucks required using the simplex method, the model is in real time, the flow rate of each road is based on the volume of material rather than the number of trucks. However, it is only appropriate when some variables are involved and it is non-negative constraints for all variables.

- Real-time Dispatching[14]: Minimizes button waste by applying the dynamic programming method, it propose a progressive time horizon when the assignment order is required, Undercarriage and over trucking conditions are taken into account, but the definition of a progressive time horizon for an assignment order is indispensable, taking into account sub-campionage and over-trucking conditions.

There are many limits of the current algorithms, namely

- The link between the strategic plan and operations: the proposed fleet management systems do not allow both short-term and long-term objectives to be achieved,

- Most models are deterministic: they assume a constant average grade for each size front and do not cover the entire life of the mine,

- The current systems do not take into account the loss of tones associated with the movement of excavators from one level to another, they present one of the main factors of deviation from the production rate,

We can identify two aspects of the fleet management system as shown in Fig. 2:

- A real-time control system using the appropriate dashboards.

- Truck loader assignment system based on algorithms \& methods.

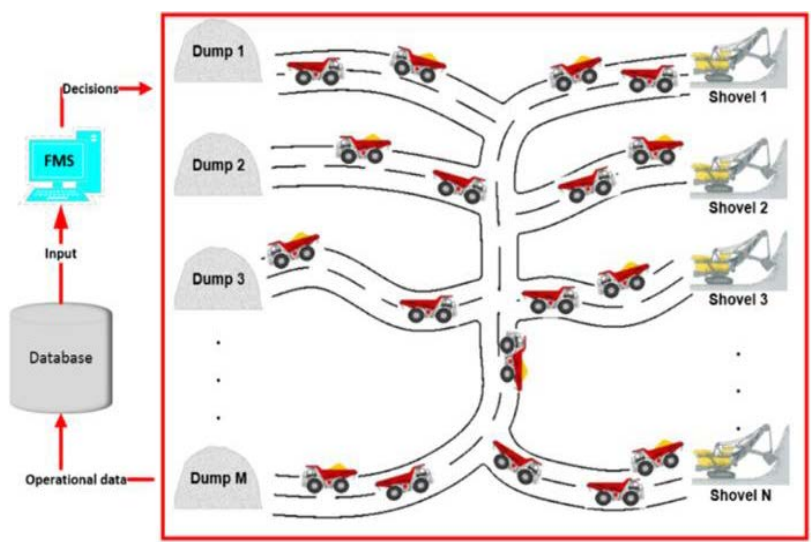

Fig. 1. Schematic of the Surface mining Operation and how it Communicates with the FMS [9].

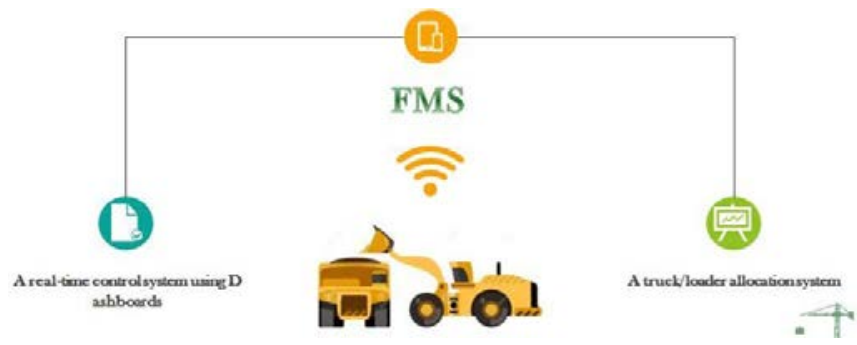

Fig. 2. Fleet Management System.

In this article, we will focus on real-time control system, with decision making Dashboards.

\section{B. Fleet Management System Evaluation}

Based on the ISA-95 standard [15], which is an international standard for control systems integration, we identify the criteria to evaluate fleet management systems as shown in Fig. 3. [17]:

ISA 95 consists of 11 production control functions[16]

- The control and resource allocation function;

- The dispatching function of production;

- The data collection and acquisition function;

- The quality management function;

- The management function of the manufacturing process;

- The planning and monitoring function;

- The performance analysis function;

- The operation management and fine-tuning scheduling function;

- The documentation management function;

- The workforce management function;

- The maintenance management function.

Nowadays, there are many companies around the world providing mining fleet management systems. Some of the most 
popular are: Modular Mining Systems, Jig-saw Software and Wenco, Dynamine TATA services Consulting [3]. However, Mi-cromine Pitram system, Viste and CAT ${ }^{\circledR}$ MineStar TM FLEET are the most common.

In our article entitled Fleet management system in real time "Stat of Art", we evaluate these industrial solutions according to ISA 95 evaluation criteria and we conclude that the majority of them do not afford:

- Management of skills and authorizations

- Dynamic resource management

- Information transmission to the different work stations Management of non-production control operations

- Observation statistical quality control and statistical process control

- Steering by automatic correction of the drifts

- Comparison with objectives

- Compliance check of files and forms

- Information Transfer between resources

\section{Artificial Intelligence for Fleet Management Systems}

Artificial intelligence (AI) is omnipresent nowadays in many technological applications (apps, websites, gaming predictions, services, etc.). It's a way to give accurate user recommendations and change its experience in many fields [18].

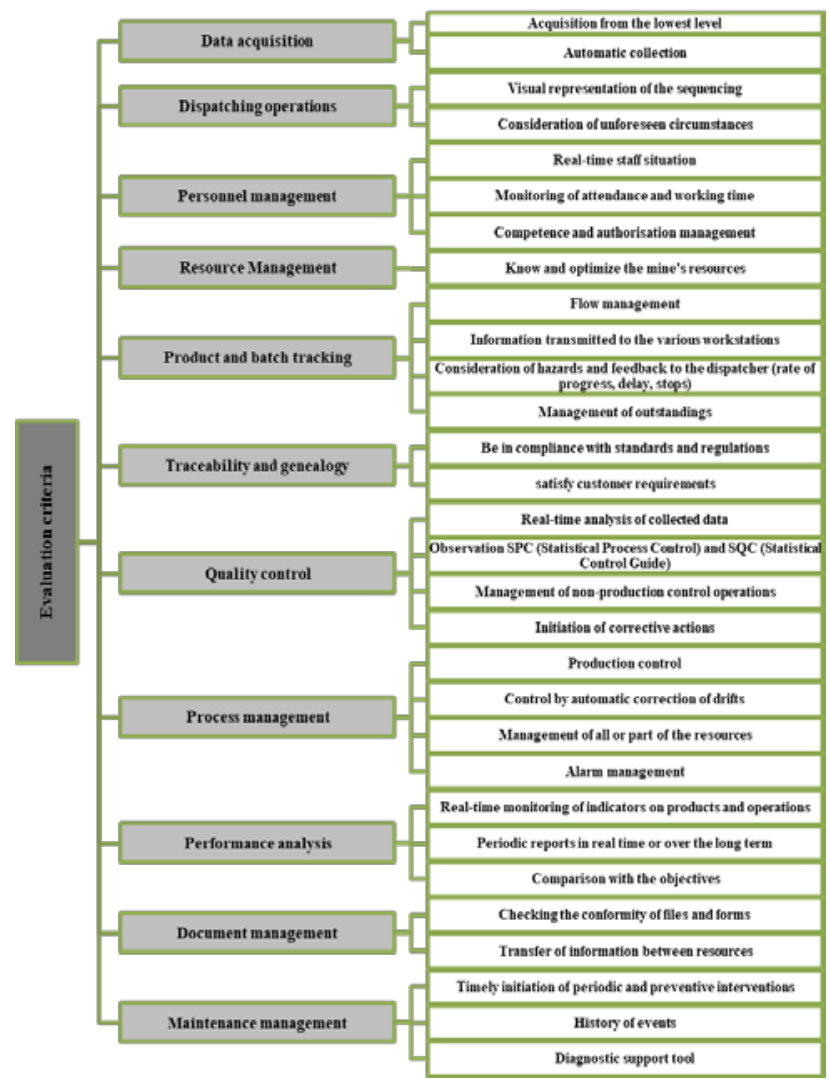

Fig. 3. The Criteria for Evaluating the Fleet Management System.
Fleet management has revolutionized AI. The priority given to commercial results and driver safety preserving cost and efficiency introduce intelligent fleet management systems.

The use of smartphones and devices recommending the best way to take is for the average driver synonym of AI presence. Drivers' lives are easier thanks to complex traffic apps that combine GPS and artificial intelligence [19]. There are many benefits of AI-based applications for fleet management such as route recommendations, road risk data analysis and driver coaching. In fact, earlier technology failed to provide the accuracy, efficiency, convenience as AI apps do. As a result, it is becoming the optimal and safer solution to transport goods and services in general and in mining field as well.

We can define AI fleet management as the use of artificial intelligence to manage fleet operations, it rationalizes fleet manager tasks by eliminating human error in transportation processes [20].

With AI recommendations in FMS, drivers, managers, and mechanics can make correct and optimal decisions to improve the fleet long-term performance. It also al-lows drivers' autonomy during each transport cycle. Table I presents some key aspects of fleet management that AI can optimize:

TABLE I. IA FOR FMS OPTIMIZATION

\begin{tabular}{|c|c|c|}
\hline IA ASPECT & USES & RESULTS \\
\hline $\begin{array}{l}\text { Real-Time Fleet } \\
\text { Analytics }\end{array}$ & $\begin{array}{l}\text { Collect data for predictive } \\
\text { analytics: } \\
\text { - traffic } \\
\text { - road conditions, } \\
\text { - environmental hazards, } \\
\text { - real-time weather, } \\
\text { - mechanical faults }\end{array}$ & $\begin{array}{l}\text { - Prioritization } \\
\text { opportunities and risks } \\
\text { - Make better routes } \\
\text { - Schedules, } \\
\text { maintenance delivery } \\
\text { - Dispatch fleet } \\
\text { outcomes and } \\
\text { activities arrangements } \\
\text { - Stay prepared for any } \\
\text { unexpected events. }\end{array}$ \\
\hline $\begin{array}{l}\text { Better Repair \& } \\
\text { Maintenance } \\
\text { Decisions }\end{array}$ & $\begin{array}{l}\text { Predictive maintenance } \\
\text { Fleets state at all times }\end{array}$ & 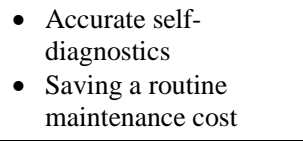 \\
\hline Fleet Integration & $\begin{array}{l}\text { - Planning, maintenance } \\
\text { and monitoring } \\
\text { operations }\end{array}$ & $\begin{array}{l}\text { - Integrating every } \\
\text { department on a single } \\
\text { platform } \\
\text { - Save time and costs } \\
\text { - Cohesive fleet } \\
\end{array}$ \\
\hline $\begin{array}{l}\text { Simpler } \\
\text { Recruitment } \\
\text { Process }\end{array}$ & $\begin{array}{l}\text { - Knowledge Management } \\
\text { - The most qualified } \\
\text { drivers' recommendation }\end{array}$ & $\begin{array}{l}\text { - Simplify the on } \\
\text { boarding process }\end{array}$ \\
\hline
\end{tabular}

\section{PROBLEMATIC}

The proposed models must be as close as possible to reality, taking into ac-count the heterogeneity of trucks and the complete modeling of an open-pit mining operation. FMS should also determine the shortest dynamic routes taking into ac-count the current location of the truck, its next destination and the time required to reach the objective in any current traffic congestion. 
Otherwise, to identify FMS users' needs and to define its main attributes in real time, a process was initiated by Zeimpekis and Vasileios [21]. The results revealed that the mining fleet management systems must meet logistics and distribution requirements such as:

- Real-time tracking of vehicle location

- Generation of vehicle and distribution system performance reports.

- Generation of proof of delivery at the end of loading and shipment.

On the other hand, we carried out within the framework of this project, a needs study within the OCP mining company (Benguerir mine), and we identified the following specifications:

- Integrated management of the production chain

- Optimization of equipment performance and elimination of losses

- Improved maintenance efficiency

- Ensure equipment security

According to the previous specifications and the evaluation of the FMS conducted in Section 2.3 and given the benefits of AI in this context, we propose a generic architecture of an Intelligent Fleet Management System for an open pit mine.

\section{Proposed Architecture}

The real-time aspect in the FMS is necessary to deal with unforeseen events. It makes it possible to detect deviations from the initial allocation plan and adjust the schedule [6] by offering immediate and efficient re-routing.

In this section, we present our fleet management proposal with a description of an FMS intelligent architecture (see Fig. 4).

The intelligent FMS architecture includes three basic layers.

IoT layer contains intelligent sensors for both autonomous and manual vehicles in the open pit mine. Every sensor is connected to an intelligent agent as software and these agents are linked to control device agent to manage them and to announce the real time status of the mine fleet for different stakeholders.

In fact, control device agent is responsible for detecting new device (new vehicle IoT sensor), update its status in FMS mapping, suspend a device and detect maintenance needs, dangers and special behaviours.).

Indeed, on each vehicle, IoT sensor is implemented and connected to open pit mine network. These sensors allow the acquisition and transmission of vehicles" information's such as the position of the machines, their speed and their conditions. They are also aware of each other's existence .One more important thing some vehicles in the mine need more intelligent sensors for specific mission or critical situations and each vehicle in the fleet is aware and reacts to the entire fleet's needs .A disruption engine determines if a fleet vehicle will be critically late for a scheduled trip and allocate alternative vehicles, as required.

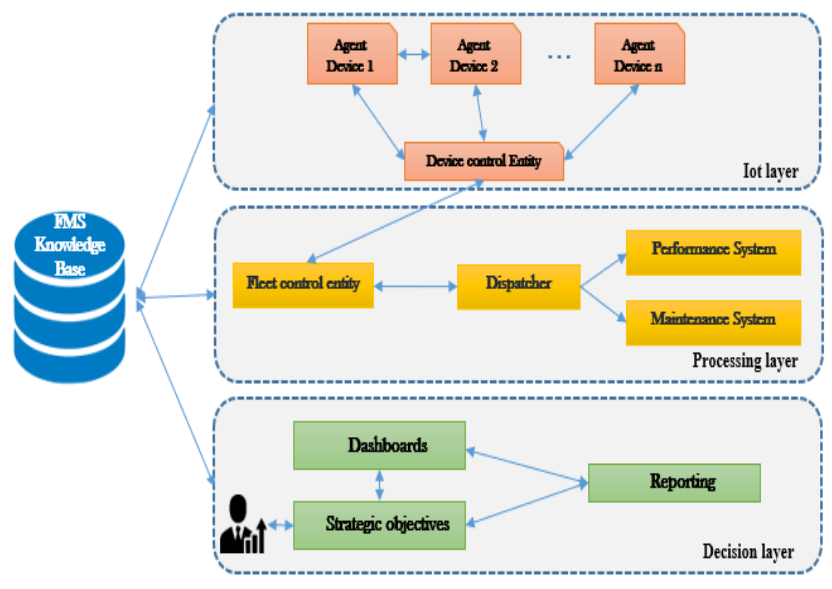

Fig. 4. Fleet Management System Architecture.

Processing layer represents the fleet coordination entities. In fact, in this level, we have:

Fleet control entity that monitors the operating states and positions of the vehicles through the communication with the device control agent. It informs stakeholders and the other agents as well of any changes in the fleet that would require an adjustment of the assignment of tasks. It has fleet reference states to be able to draw up a real-time, vigilant and continuous comparison.

Dispatcher Agent: once executed, this entity recalculates the overall allocation of tasks for mining vehicles. It has two dispatching algorithms based on a set of assignment criteria: Short-term allocation algorithm and a long-term allocation algorithm. The dispatcher is one of the most intelligent entities in our FMS. It is up to it to de-fine the mining missions by taking into consideration the strategic decisions and the real state of the fleet. Dispatcher agent also executes optimized dispatching in collaboration with performance and maintenance systems to research fleet vehicle that should go next and in which itinerary.

Maintenance system manages vehicles under maintenance. It allows updating their status and gives information about what is wrong with them. It also helps drivers and open pit mine responsible (s) to have real time informations about vehicles maintenance status. It is the necessary data to make dispatching allocation strategies (long term and short term).

Performance system: It is the intelligent system that ensures FMS performance management, through machine learning models. The datasets are made from real and successful open pit mine missions in OCP. This system $y$ is connected to the knowledge base just like all the entities of the processing layer.

Decision layer it is the layer which mainly interfaces the FMS with its potential users (directors and executive managers) via:

- Collecting the strategic objectives of the open pit mine from top management and sending them in 
synchronous or asynchronous mode to the dispatcher to develop the mining missions.

- Display of performance indicators and real-time and instantaneous graphs representing the state of the mine (production and fleet).

Decision layer is also able to generate stats and administrative documents in order to facilitate mining regulations respect.

\section{IMPLEMENTATION}

As for expected tools for the proposed intelligent fleet management system, we use mainly Internet of Things (IoT) technology.

IoT refers to a network of actuators and sensors continuously collecting data from IoT is a network of sensors continuously collecting environmental data. In the open pit fleet management system, the IoT collects data for analysis. It ensures the transparent sharing of information between all stakeholders in the supply chain, such as drivers, production managers and dispatchers.

For our intelligent fleet management system, we use the three main technologies of IoT:

- Wireless communication (4G, Bluetooth, WiFi) to transmit the relevant information between the different vehicles of the mine.

- Global positioning system (GPS) for precise location tracking in real time.

- Integrated diagnostic analyzers (such as OBDII and J1939) for self-diagnosis and reporting

The smart FMS also has cameras to ensure that video data can be captured, analyzed and accessible at all times, allowing better study of driver behaviour, road conditions or hazards. All the more, machine learning technology allows fleets to learn data collected over time and make managed adjustments based on that data in the performance management entity.

The proposed intelligent FMS with all the components and technologies used is able to perform the following tasks:

- Collection of precise mining transport data and its transmission to other devices.

- Transmission of information at all levels of the supply chain from top management to implementers.

- Analyze data in real time and advice drivers on the best course of action.

- Performing self-diagnostics of mining vehicles and recommending solutions via predictive maintenance.

This is important because it creates a new era of mining fleet management system in which human errors are reduced and open pit mine production is optimized in different aspects of the transport cycle. This led to better results and cost optimization. Fig. 5 presents the first version of open pit mine platform dashboards. The platform covers ISA95 main domains.

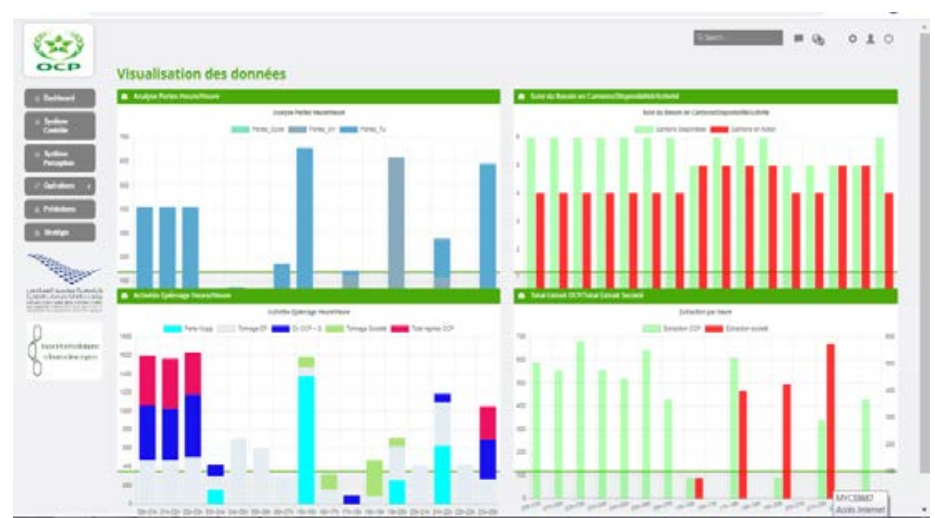

Fig. 5. The First Version of Open Pit Mine Platform Dashboards.

Primary results were positively validated and appreciated by experiment mine actors.

\section{CONCLUSION AND PERSPECTIVES}

In this article, we proposed an intelligent fleet management system focusing on re-al-time management of mining equipment by applying a multi-agent system. First, we presented a state of the art of fleet management systems as well as some definitions of the multi-agent system. In addition, we identified the criteria for evaluating a fleet management system based on the ISA95 standard and finally we proposed architecture for an intelligent fleet management system composed of three layers: the vehicle layer, the processing layer and the decision layer. We implemented the first version of this architecture and test it in Benguerir experimental mine in order to improve it and extend its application scope.

In perspective, we will evaluate the proposed system using the criteria of the ISA95 standard and improve dispatching algorithms; we will also detail and implement every part of the smart fleet management system architecture.

\section{ACKNOWLEDGMENT}

This research was supported by Mohammed VI Polytechnic University de Benguerir, Morocco and OCP group. We thank our colleagues from university and from OCP group who provided insight and expertise that greatly assisted the research.

REFERENCES

[1] Ch. Achillas, D. Bochtis, D. Aidonis, V. Marinoudi, and D. Folinas, "Voice-driven fleet management system for agricultural operations," Inf. Process. Agric., vol. 6, no. 4, pp. 471-478, Dec. 2019, doi: 10.1016/j.inpa.2019.03.001.

[2] E. Krelja Kurelović and B. Škabić, "Comparison of fleet management systems," Zb. Veleuč. U Rijeci, vol. 6, no. 1, pp. 357-370, 2018, doi: 10.31784/zvr.6.1.27.

[3] A. M. Afrapoli and H. Askari-Nasab, "Mining fleet management systems: a review of models and algorithms," Int. J. Min. Reclam. Environ., vol. 33, no. 1, pp. 42-60, Jan. 2019, doi: 10.1080/17480930.2017.1336607.

[4] "Wellman - (54) FLEET MANAGEMENT SYSTEM.pdf."

[5] P. Chaowasakoo, H. Seppälä, H. Koivo, and Q. Zhou, "Improving fleet management in mines: The benefit of heterogeneous match factor," Eur. J. Oper. Res., vol. 261, no. 3, pp. 1052-1065, Sep. 2017, doi: 10.1016/j.ejor.2017.02.039. 
[6] H. Billhardt et al., "Dynamic Coordination in Fleet Management Systems: Toward Smart Cyber Fleets,” IEEE Intell. Syst., vol. 29, no. 3, pp. 70-76, May 2014, doi: 10.1109/MIS.2014.41.

[7] H. Yamamoto and R. B. Ramli, "Real-time Decision Making of Agents to Realize Decentralized Autonomous FMS by Anticipation,” p. 11, 2006.

[8] S. Parhi and S. C. Srivastava, "A Highly Efficient and Optimised Simulation Based Multi objective Decision-Making for FMS Control," Int. J. Emerg. Res. Manag. Technol., vol. 6, no. 6, p. 98, Jun. 2018, doi: 10.23956/ijermt.v6i6.254.

[9] A. Moradi Afrapoli, M. Tabesh, and H. Askari-Nasab, "A multiple objective transportation problem approach to dynamic truck dispatching in surface mines,” Eur. J. Oper. Res., vol. 276, no. 1, pp. 331-342, Jul. 2019, doi: 10.1016/j.ejor.2019.01.008.

[10] A. W. I. S. | www.anchorwave.com, "DISPATCH Fleet Management System | Modular Mining Systems, Inc.” https://www.modularmining .com/product/dispatch/ (accessed Mar. 16, 2019).

[11] “Modular Mining | MineSmarterTM,” Modular Mining. https://www. modularmining.com/ (accessed Jun. 13, 2019).

[12] M. Najafi, R. Rafiee, and S. M. E. Jalali, "Open pit limit optimization using dijkstra's algorithm,” p. 5, 2020.

[13] Shiv PHoomari-Nasaban Ask and Shiv Prakash Upadhyay, "Dynamic shovel allocation approach to short-term production planning in open-pit mines,” International Journal of Mining, Reclamation and Environment, 2017.

[14] R. B. Dannenberg, "Time-Flow Concepts and Architectures For Music and Media Synchronization,” p. 6.
[15] L. Yue, P. Niu, and Y. Wang, "Guidelines for defining user requirement specifications (URS) of manufacturing execution system (MES) based on ISA-95 standard,” J. Phys. Conf. Ser., vol. 1168, p. 032065, Feb. 2019, doi: 10.1088/1742-6596/1168/3/032065.

[16] “Le standard ISA 95 - Smart Manufacturing,” TLG Pro, Feb. 05, 2018. https://www.tlgpro.fr/2018/02/standard-isa-95/ (accessed Jan. 02, 2019).

[17] B. Scholten, The road to integration: a guide to applying the ISA-95 standard in manufacturing. Research Triangle Park NC: ISA, 2007.

[18] M. Chergui, H. Medromi, and A. Sayouti, "Inter-organizational Workflow for Intelligent Audit of Information Technologies in terms of Entreprise Business Processes,” Int. J. Adv. Comput. Sci. Appl., vol. 5, no. 5, 2014, doi: 10.14569/IJACSA.2014.050515.

[19] A. M. Nascimento et al., "A Systematic Literature Review About the Impact of Artificial Intelligence on Autonomous Vehicle Safety,” IEEE Trans. Intell. Transp. Syst., pp. 1-19, 2019, doi: 10.1109/TITS.2019.2949915.

[20] R. Salazar-Cabrera, A. P. De La Cruz, and J. M. Madrid Molina, "Fleet Management and Control System from Intelligent Transportation Systems perspective," in 2019 2nd Latin American Conference on Intelligent Transportation Systems (ITS LATAM), Bogota, Colombia, Mar. 2019, pp. 1-7, doi: 10.1109/ITSLATAM.2019.8721347.

[21] V. Zeimpekis, G. M. Giaglis, and I. Minis, "Development and Evaluation of an Intelligent Fleet Management System for City Logistics," in Proceedings of the 41st Annual Hawaii International Conference on System Sciences (HICSS 2008), Waikoloa, HI, USA, Jan. 2008, pp. 72-72, doi: 10.1109/HICSS.2008.121. 